ARTIGO ORIGINAL

Revista de Enfermagem do Centro-Oeste Mineiro

2021; 11/4301

DOI: http://doi.org/10.19175/recom.v11i0.4301

www.ufsj.edu.br/recom

\title{
Processo de enfermagem no ambiente hospitalar: potencialidades, fragilidades e estratégias vivenciadas por enfermeiros
}

\author{
Nursing process in the hospital environment: potentialities, fragilities and strategies experienced by \\ nurses
}

\author{
Proceso de enfermería en el entorno hospitalario: potencialidades, fragilidades y estrategias \\ experimentadas por los enfermeros
}

\section{RESUMO}

Objetivo: Descrever as potencialidades e fragilidades vivenciadas por enfermeiros, atuantes no contexto hospitalar acerca do Processo de Enfermagem, bem como as estratégias para auxiliar nesse contexto. Método: Pesquisa exploratória, descritiva, de abordagem qualitativa, realizada com 15 enfermeiros atuantes em um hospital público municipal, localizado no Rio Grande do Sul, Brasil. Os dados foram coletados por meio de um questionário semiestruturado, os quais foram submetidos à análise textual discursiva. Resultados: Geraram uma categoria central - vivências de enfermeiros, atuantes no contexto hospitalar acerca do Processo de Enfermagem; e oito categorias, das quais, três referentes a unidade de base - potencialidades vivenciadas por enfermeiros acerca do Processo de Enfermagem; três, referentes a unidade fragilidades vivenciadas por enfermeiros acerca do Processo de Enfermagem; e duas, sobre a unidade - Estratégias sugeridas para auxiliar na realização do Processo de Enfermagem. Conclusão: A compreensão das potencialidades e fragilidades é fundamental para o planejamento de estratégias para auxiliar no processo de enfermagem.

Descritores: Serviço Hospitalar de Admissão de Pacientes; Gestão em Saúde; Cuidado de Enfermagem; Processo de Enfermagem.

\section{ABSTRACT}

Objective: To describe the strengths and weaknesses experienced by nurses, working in the hospital context about the Nursing Process, as well as the strategies to assist in this context. Method: Exploratory, descriptive research with a qualitative approach, conducted with 15 nurses working in a municipal public hospital, located in Rio Grande do Sul, Brazil. Data were collected through a semi-structured questionnaire, which were subjected to discursive textual analysis. Results: It generated a central category - nurses' experiences, working in the hospital context about the Nursing Process; and nine categories, of which, three referring to the basic unit - potentialities experienced by nurses about the Nursing Process; three, referring to the unit - weaknesses experienced by nurses about the Nursing Process; and two, on the unit - Strategies suggested to assist in the realization of the Nursing Process. Conclusion: Understanding the strengths and weaknesses is essential for planning strategies to assist in the nursing process.

Descriptors: Hospital service for patient admission; Health management; Nursing care; Nursing process.

\section{RESUMEN}

Objetivo: Describir las fortalezas y debilidades vividas por los enfermeros, trabajando en el contexto hospitalario sobre el Proceso de Enfermería, así como las estrategias para ayudar en este contexto. Método: Investigación exploratoria descriptiva con enfoque cualitativo, realizada con 15 enfermeros que laboran en un hospital público municipal, situado en Rio Grande do Sul, Brasil. Los datos fueron recolectados a través de un cuestionario semiestructurado, los cuales fueron sometidos a análisis discursivo textual. Resultados: Generó una categoría central - experiencias de enfermeros, trabajando en el contexto hospitalario sobre el Proceso de Enfermería; y nueve categorías, de las cuales tres se refieren a la unidad básica - potencialidades vividas por los enfermeros sobre el Proceso de Enfermería; tres, referido a la unidad - debilidades experimentadas por los enfermeros sobre el Proceso de Enfermería; y dos, en la unidad - Estrategias sugeridas para ayudar en la realización del Proceso de Enfermería. Conclusión: Comprender las fortalezas y debilidades es fundamental para planificar estrategias que ayuden en el proceso de enfermería.

Descriptores: Servicio hospitalario de ingreso de pacientes; Manejo de la salud; Cuidado de enfermera; Proceso de enfermería.

Raysa Fernandes Moreira ${ }^{1}$
$\underline{0000-0003-0941-5467}$
Bethânia Kraemer Haag ${ }^{2}$
$\underline{0000-0002-1766-217 X}$
Cláudia Zamberlan ${ }^{1}$
$\underline{0000-0002-4664-0666}$
Rosiane Filipin Rangel
$\underline{0000-0003-4059-4176}$
Silomar Ilha ${ }^{1}$
$\underline{0000-0002-2132-9505}$

${ }^{1}$ Universidade Franciscana, Brasil ${ }^{2}$ Hospital Geral da UNIMED, Brasil

Autor correspondente: Silomar llha E-mail: silo_sm@hotmail.com

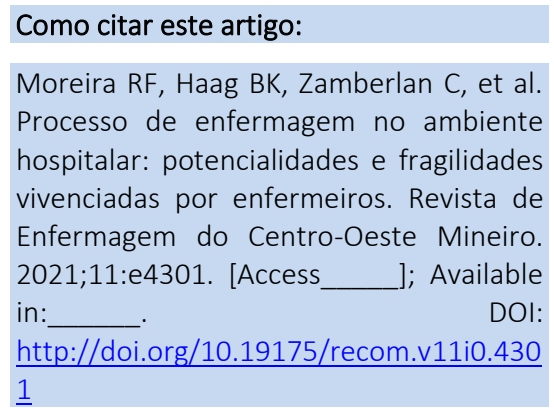




\section{INTRODUÇÃO}

A sistematização da Assistência de Enfermagem (SAE) caracteriza-se como uma especificidade no processo de cuidar e, para que se torne efetiva, o enfermeiro deve primeiramente conhecer cada paciente e assim planejar um plano de cuidados individualizado. Dessa forma, pode se identificar situações de saúde/doença e promover a promoção, prevenção, recuperação e reabilitação da saúde do indivíduo, da família e da comunidade, proporcionando a valorização das suas necessidades, conduzida por meio do cuidado e da eficiência nas intervenções ${ }^{(1)}$.

O Processo de Enfermagem (PE), por sua vez, é parte integrante da SAE e pode ser definido como um instrumento metodológico para organizar a assistência aos pacientes, uma vez que este viabiliza a autonomia profissional a partir da sua execução completa, respeitando a sequência de suas etapas ${ }^{(2)}$, quais sejam: coleta de dados de enfermagem ou histórico de enfermagem; diagnósticos de enfermagem; planejamento de enfermagem, implementação e avaliação de enfermagem ${ }^{(3)}$. Quando utilizado de maneira adequada, O PE favorece as ações a serem desenvolvidas evitando maior demanda de trabalho, pois direciona a tomada de decisão dos profissionais, resultando positivamente no cuidado, o qual pode ser novamente planejado se necessário ${ }^{(2)}$.

No que se refere ao cuidado ao ser humano hospitalizado, o mesmo é complexo, pois a pessoa nessa condição, pode tornar-se vulnerável e com limitação da independência resultante, dentre tantos aspectos, da condição clínica em que a mesma se encontra e do próprio contexto hospitalar, configurado pelas suas normas, rotinas e protocolos institucionais. No hospital, ao vivenciar o adoecimento e o período de tratamento, as pessoas começam a conviver com outras pessoas internadas, as quais não faziam parte da sua rotina diária, o que denota mudanças do seu cotidiano de vida(4).

Assim, o período de internação e a doença são experenciados de maneira intensa, ultrapassando, algumas vezes, a dimensão emocional, podendo emergir respostas físicas, resultante dos sentimentos que foram surgindo no decorrer do processo. As famílias que vivenciam o adoecer de seus entes queridos, tornam-se, por vezes, igualmente vulneráveis devido a insegurança trazida pela doença, o que pode acarretar alterações do estado emocional tanto da pessoa internada, quanto do acompanhante/familiar ${ }^{(5)}$.

Dessa forma, percebe-se a necessidade dos profissionais da saúde, em especial do enfermeiro, por ser o responsável pela SAE, estabelecer condutas que permitam o vínculo com a pessoa hospitalizada e com o acompanhante/familiar. O vínculo pode ser compreendido como um componente facilitador e desafiador, constituindo-se em um forte elo entre o profissional de saúde e o paciente, com vistas à garantia da segurança no atendimento de suas necessidades, promovendo uma relação de respeito e confiança com o paciente e com a sua família(6).

É por meio da comunicação e do vínculo criado com essas famílias que os enfermeiros conseguem desenvolver uma relação de maior proximidade, conhecer melhor as suas necessidades e conquistar a confiança das pessoas $^{(7)}$. Dessa forma, o enfermeiro possui maior possibilidade de desenvolver a SAE e O PE coerente com as necessidades das pessoas e famílias.

A Resolução do Conselho Federal de Enfermagem - COFEN 358/2009 dispõe sobre a implementação do PE em todos os ambientes, sejam eles públicos ou privados, no qual ocorre o cuidado de enfermagem, devendo ser registrada formalmente a execução do $\mathrm{PE}$, pois auxilia na documentação do exercício profissional ${ }^{(3)}$. Contudo, percebe-se que embora O PE seja previsto por uma resolução e obrigatório em todos os contextos de atuação dos profissionais de enfermagem, ainda há lacunas no que diz respeito a efetivação do mesmo como potencial de transformação. $O$ que se percebe é que $O P E$ no contexto hospitalar tem sido desenvolvido ancorado na fiscalização e na obrigatoriedade, e não pela compreensão e singularização de sua necessidade e relevância por parte de alguns profissionais. Assim, torna-se necessário conhecer as vivências de enfermeiros nesse processo, fato que justifica a necessidade e a relevância dessa pesquisa.

Frente ao exposto questiona-se: Quais são as potencialidades e fragilidades vivenciadas por enfermeiros no contexto hospitalar acerca do Processo de Enfermagem? Que estratégias podem ser pensadas para auxiliar nesse contexto? $\mathrm{Na}$ tentativa de responder os questionamentos, objetivou-se: descrever as potencialidades e fragilidades vivenciadas por enfermeiros, atuantes no contexto hospitalar acerca do Processo de 
Enfermagem, bem como as estratégias para auxiliar nesse contexto.

\section{MÉTODOS}

Trata-se de uma pesquisa exploratória, descritiva, de abordagem qualitativa, realizada com enfermeiros atuantes em um hospital público municipal, de médio porte localizado no Rio Grande do Sul, Brasil. O referido hospital contava, no momento da pesquisa, com oito unidades assistenciais, com as seguintes especialidades: ginecologia, obstetrícia, mastologia, nefrologia, oftalmologia, ortopedia, pediatria, otorrinolaringologia, pneumologia, psiquiatria, urologia, nutrição, hematologia, gastroenterologia, cirurgia geral, psicologia, fonoaudiologia, clínica médica, dermatologia, angiologia e cirurgia vascular. Havia aproximadamente 112 leitos, contendo em torno de 300 profissionais, sendo 22 enfermeiros.

A escolha do cenário do estudo ocorreu por ser um hospital que serve de campo de prática para estudantes de enfermagem, bem como de outras áreas da saúde. Ainda pelo fato de que o mesmo estava sendo ampliado e, consequentemente, resultará na necessidade de contratação de um maior número de profissionais de diferentes áreas de atuação, especialmente da equipe de enfermagem, considerada o maior quadro de efetivo do hospital. Salienta-se que a instituição possuía o processo de enfermagem estruturado, informatizado e empregavam como padronização a taxonomia da North American Nursing Diagnosis Association (NANDA) ${ }^{(8)}$.

Os dados foram coletados, no mês de novembro do ano de 2020, por uma das pesquisadoras, a qual foi capacitada junto ao núcleo de pesquisa para a coleta. Além disso, a mesma já havia desenvolvido estudos e pesquisas sobre a temática. Para a coleta, utilizou-se um questionário semiestruturado construído especialmente para essa pesquisa, o qual foi estruturado em duas partes. Inicialmente buscouse a caracterização dos participantes e posteriormente elaborou-se seis questões abertas a fim de contemplar o objetivo proposto. Para a entrega dos questionários, inicialmente, a pesquisadora principal junto à Gerência de Enfermagem, teve posse da escala de trabalho contendo informações sobre o nome e setor de todos os profissionais enfermeiros.

Com a escala, a pesquisadora principal realizou o convite individual pessoalmente, durante uma visita às unidades. Conforme aceite dos mesmos, foi entregue o questionário semiestruturado, envolto em saco plástico após higienizá-lo com álcool 70\%, tendo em vista as precauções vivenciadas em detrimento à pandemia causada pela COVID-19. Embora fosse um momento sensível vivenciado pelo serviço de saúde, a pandemia não interferiu na execução do PE no cenário do estudo.

Como critérios de inclusão da pesquisa, considerou-se: os profissionais enfermeiros e os residentes enfermeiros, com atuação mínima de seis meses no referido hospital. Esse período mínimo de atuação foi estipulado, por compreender que seja tempo suficiente para que os mesmos já tenham vivenciado a rotina do ambiente hospitalar, bem como as questões relacionadas ao $\mathrm{PE}$, estando aptos para responderem aos questionamentos. Foram considerados excluídos da pesquisa, os enfermeiros e ou residentes de enfermagem que estavam de férias, atestado ou licença maternidade no período da coleta de dados. Quatro profissionais possuíam menos de seis meses de atuação e três estavam de férias no período de coleta dos dados. Com base nisso, atenderam aos critérios de inclusão, formando o corpus dessa pesquisa 15 profissionais.

O tratamento dos dados foi realizado por dois pesquisadores concomitantemente, com base na técnica da análise textual discursiva, organizada a partir de uma sequência recursiva de três componentes: 1) unitarização; 2) estabelecimento de relações; 3) comunicação(9). Inicialmente os pesquisadores examinaram com intensidade e profundidade os textos em detalhes, formando a categoria central, a partir da identificação das vivências de enfermeiros atuantes no contexto hospitalar acerca do PE. A mesma foi unitarizada em três unidades de base; na primeira unidade, agruparam-se todas as potencialidades vivenciadas por enfermeiros acerca do PE; na segunda, as fragilidades vivenciadas por enfermeiros acerca do PE; e na terceira, as estratégias sugeridas para auxiliar na realização do PE.

Após, foi realizada nova leitura a partir da categoria central e das unidades de base, buscando o estabelecimento de relações entre elas, ou seja, cada relato inserido nas unidades de base foi lido de forma minuciosa, sendo separados em diferentes unidades. Por fim, procedeu-se a última etapa do método de análise, onde o pesquisador apresentou as compreensões atingidas a partir dos dois focos anteriores, pelo processo de comunicação entre as diferentes dificuldades, resultando nos metatextos de 
descrição e interpretação dos fenômenos investigados, dando origem as categorias.

Foram considerados os preceitos éticos que envolvem a pesquisa com seres humanos, conforme a Resolução 466/2012 do Ministério da Saúde ${ }^{(10)}$. O projeto foi aprovado por Comitê de Ética em Pesquisa pelo parecer número: 4.390.680 e CAAE: 38657120.4.0000.5306. Os participantes foram identificados pelas letras $E$ (Enfermeiro) seguidas de um algarismo numérico (E1, E2...E15).

\section{RESULTADOS}

Inicialmente apresenta-se o Quadro 1 a caracterização dos participantes da pesquisa, sendo descritos os achados com relação ao número de participantes, sexo, área de especialidade para os enfermeiros do quadro permanente e área do curso em andamento para os residentes, o tempo de experiência profissional e de atuação no serviço.

Quadro 1. Caracterização dos participantes. Rio Grande do Sul, Brasil, 2020.

\begin{tabular}{|c|c|c|c|c|c|}
\hline \multirow{2}{*}{$\begin{array}{l}\text { Participantes/ } \\
\text { Número } \\
11\end{array}$} & \multicolumn{2}{|c|}{ Sexo } & \multirow{2}{*}{$\begin{array}{l}\text { Especialidade/área } \\
\qquad 9\end{array}$} & \multirow{2}{*}{$\begin{array}{c}\text { Tempo de } \\
\text { experiência } \\
\text { profissional } \\
\text { em anos }\end{array}$} & \multirow{2}{*}{$\begin{array}{l}\text { Tempo de } \\
\text { atuação no } \\
\text { serviço } \\
\text { em anos }\end{array}$} \\
\hline & $\mathrm{F}$ & M & & & \\
\hline \multirow{5}{*}{$\begin{array}{l}\text { Enfermeiros do } \\
\text { quadro } \\
\text { permanente do } \\
\text { cenário }\end{array}$} & \multirow{5}{*}{9} & \multirow{5}{*}{2} & 5- Enfermagem obstétrica & \multirow{5}{*}{1 a 29} & \multirow{5}{*}{1 a 8} \\
\hline & & & 1 Infectologia e neurologia & & \\
\hline & & & 1 Enfermagem na Urgência e Trauma & & \\
\hline & & & 1 Oncologia & & \\
\hline & & & 1 Estratégia de Saúde da Família & & \\
\hline \multirow{2}{*}{$\begin{array}{l}\text { Participantes/ } \\
\text { Número } \\
4\end{array}$} & \multicolumn{2}{|c|}{ Sexo } & & \multirow{2}{*}{$\begin{array}{l}\text { Tempo de } \\
\text { experiência } \\
\text { profissional } \\
\text { em meses }\end{array}$} & \multirow{2}{*}{$\begin{array}{l}\text { Tempo de } \\
\text { atuação no } \\
\text { serviço } \\
\text { em meses }\end{array}$} \\
\hline & $\mathrm{F}$ & M & Área da residência em andamento & & \\
\hline $\begin{array}{l}\text { Enfermeiros } \\
\text { residentes no } \\
\text { cenário }\end{array}$ & 4 & 0 & $\begin{array}{l}2 \text { - Infectologia e neurologia } \\
\text { 2- Enfermagem obstétrica }\end{array}$ & 9 & 9 \\
\hline
\end{tabular}

Os dados analisados resultaram em uma categoria central: vivências de enfermeiros, atuantes no contexto hospitalar acerca do Processo de Enfermagem. A mesma foi unitarizada em três unidades de base: Potencialidades vivenciadas por enfermeiros acerca do Processo de Enfermagem; Fragilidades vivenciadas por enfermeiros acerca do Processo de Enfermagem; Estratégias sugeridas para auxiliar a realização do Processo de Enfermagem. Tais unidades geraram oito categorias, conforme demonstrado da Figura 1.

Figura 1 - Representação esquemática da relação entre a categoria central, unidades de base e as categorias.

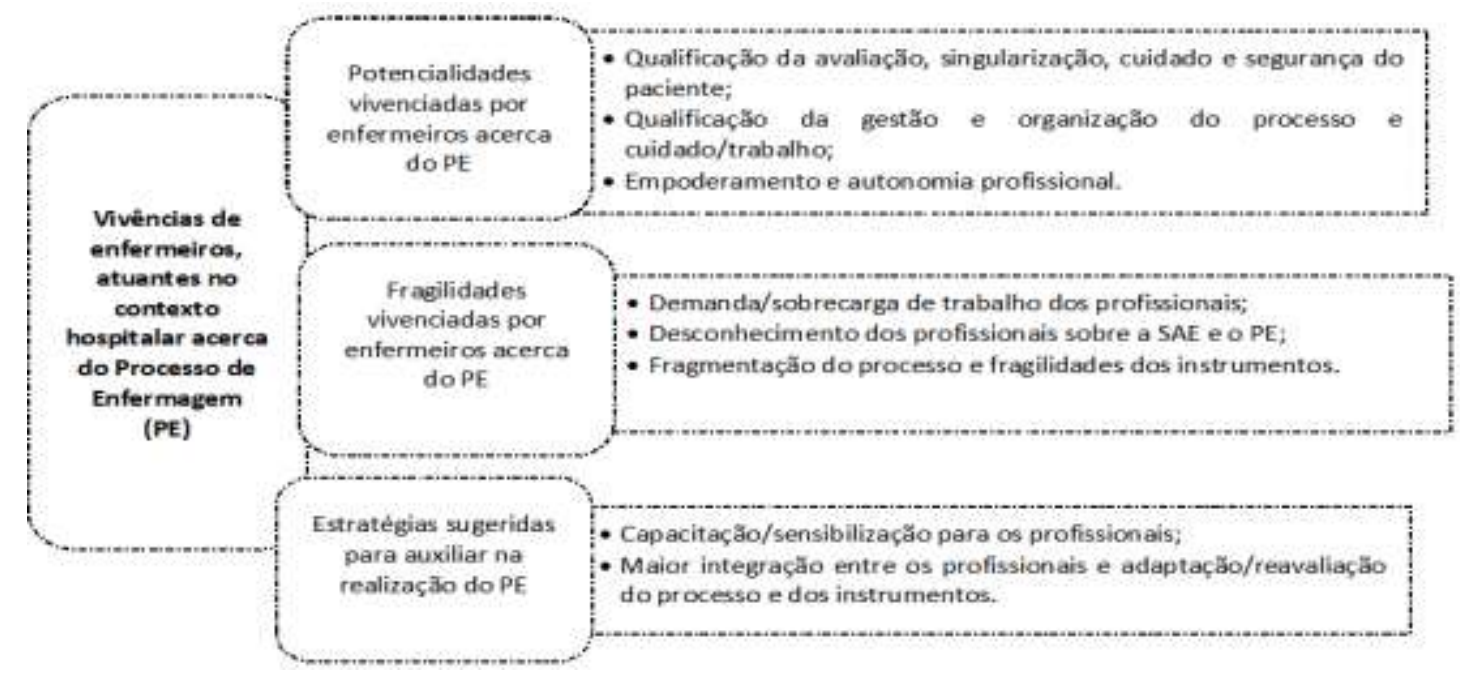

Fonte: dados da pesquisa, 2020. 
Uma das unidades que se destacou, versa sobre as Potencialidades vivenciadas por enfermeiros acerca do $\mathrm{PE}$, por meio de três categorias: qualificação da avaliação, singularização, cuidado e segurança do paciente; qualificação da gestão e organização do processo e cuidado/trabalho; e empoderamento e autonomia profissional.

\section{Qualificação da avaliação, singularização, cuidado e segurança do paciente}

Uma das potencialidades vivenciadas pelos enfermeiros no contexto hospitalar, acerca do PE, versa sobre a contribuição deste para a avaliação singular de cada paciente, o que contribui para a qualidade do cuidado e segurança do paciente, conforme pode ser visualizado nos relatos: "Sim, facilita principalmente ver o paciente como um todo" (E1). "Com as etapas do Processo de Enfermagem conseguimos avaliar e reavaliar 0 paciente diariamente, pois obriga o enfermeiro a olhar com cuidado cada paciente" (E2). "Auxilia na melhor qualidade da assistência prestada" (E3). "Oportuniza visualizar e avaliar a singularidade de cada paciente; conhecer o paciente, sua história, criar vínculo para boa assistência é de extrema relevância, dado que cada paciente tem sua singularidade, portanto devemos avaliar cada um levando em conta seu histórico. Acaba por favorecer a assistência prestada" (E4). "Sim, garantir que as intervenções sejam voltadas para o indivíduo e não a doença, evitar erro na assistência. Reduzir o tempo de internação, promovendo a recuperação" (E6). "Sim, a identificação de achados clínicos, organização do cuidado e adequação do atendimento é importante para otimizar atendimento e prestação do cuidado humanizado conforme as individualidades" (E7). "Sim, conduz a uma assistência de qualidade; Segurança; Gerenciamento da enfermagem; Fortalecimento do trabalho em equipe, facilita na recuperação do paciente e na prevenção de agravos. Importantíssima, devido proporcionar um conhecimento integral do paciente, e maior segurança tanto para o paciente como na assistência realizada como também promover uma avaliação" (E8). "O Processo de Enfermagem facilita a assistência, tendo em vista que as etapas conseguem contemplar o paciente na sua totalidade, não focando somente no diagnóstico da doença [...]" (E9). "A partir do processo de enfermagem o enfermeiro, muitas vezes, tem a visão em âmbito geral sobre as fragilidades do paciente e a partir disso, elabora os diagnósticos, buscando oferecer um cuidado mais humanizado. Proporciona, assim, um cuidado humanizado e com qualidade" (E13).

Observa-se ainda, que o PE na percepção dos enfermeiros, contribui para o fortalecimento do vínculo entre profissional e paciente, uma vez que potencializa intervenções voltadas ao ser humano e não à doença. Além disso, os enfermeiros compreendem que o PE conduz ao gerenciamento da enfermagem e ao fortalecimento do trabalho em equipe.

\section{Qualificação da gestão e organização do processo e cuidado/trabalho}

A qualificação da gestão e a organização do processo, tanto no que se refere ao cuidado, como ao trabalho também são vistos pelos enfermeiros como uma potencialidade da utilização do PE no contexto hospitalar: "As principais potencialidades, ao meu ver, são a organização da assistência, de forma coordenada e continua, a avaliação desta assistência se está sendo adequada as necessidades do paciente, além de trazer benefícios a instituição no processo de auditoria" (E5). "[...] elabora o processo referente a alta hospitalar, planejando uma melhora clínica mais ágil" (E9). "Gestão, organização, individualização e humanização na assistência" (E12). "Percebe-se a SAE como valioso instrumento de início de acolhimento e planejamento das ações e intervenções a serem propostas ao paciente e sua família" (E6). "A SAE será percebida a partir da admissão do paciente, a organização do serviço e tudo que faz parte do cuidado e o processo é a elaboração dos cuidados" (E13).

Pode se perceber por meio do relato do participante E5 que além dos benefícios ao paciente o PE contribui para o serviço, uma vez que auxilia no processo de auditoria. E6, por sua vez, amplia a contribuição do PE no que se refere ao aspecto do cuidado, pois traz em seus descritos a relevância do mesmo, tanto ao paciente quanto para a família. Já E13 apresenta uma diferenciação no que se refere a SAE e o PE, quando descreve que a SAE diz respeito ao aspecto organizacional do enfermeiro para que o cuidado possa ocorrer, enquanto o PE é, de fato, a elaboração dos cuidados.

\section{Empoderamento e autonomia profissional}

Além da qualificação do cuidado e da gestão, o PE contribui para o empoderamento e à autonomia profissional do enfermeiro, conforme 
os relatos: "O processo de Enfermagem contribui para o empoderamento do enfermeiro" (E10). "O Processo de Enfermagem demonstra o papel ampliado do enfermeiro, a sua percepção, autonomia e a humanização" (E11).

Pode se perceber, ainda, que O PE conforme o participante E11, contribui para a visualização da ampliação da atuação do enfermeiro, pois por meio dele, o profissional traz a sua percepção, demonstra a sua autonomia e reforça a humanização. Contudo, as fragilidades vivenciadas por enfermeiros acerca do PE, também se destacaram como uma unidade de base, por meio de três categorias: demanda/sobrecarga de trabalho dos profissionais; desconhecimento dos profissionais sobre a SAE e o PE; e fragmentação do processo e fragilidades dos instrumentos.

\section{Demanda/sobrecarga de trabalho dos profissionais}

Uma das fragilidades apontadas pelos enfermeiros na realização do PE ocorre devido à rotina intensa, que leva a grande demanda e sobrecarga de trabalho dos profissionais/equipe de enfermagem, não conseguindo ser realizado em sua totalidade como deveria: "A rotina diária de uma unidade hospitalar clínica é intensa e corrida, muitas vezes fazendo com que os profissionais não sigam devidamente todas as etapas do Processo de Enfermagem" (E2). "Dificuldade da equipe técnica seguir a prescrição de enfermagem com entendimento" (E3). "Demanda da unidade e a falta de profissionais empenhados em realizar a sistematização da assistência" (E4). "Irrelevância vista pela equipe multidisciplinar, sobrecarga profissional dificultando realizar as etapas" (E7). "[...] déficit de funcionários" (E10). "Muitas vezes o que você planeja para a implementação não consegue desenvolver, seja por sobrecarga de trabalho, pouco tempo para desenvolver os cuidados, falta de recursos" (E13).

Os relatos evidenciam, também, que os enfermeiros sentem a falta de empenho de alguns profissionais para a realização da SAE, bem como pouca importância demonstrada pela equipe multidisciplinar sobre a sua efetivação. Relatam ainda que o déficit de funcionários, somados a outras fragilidades, impossibilitam o desenvolvimento das ações planejadas.

Desconhecimento dos profissionais sobre a SAE e OPE

O desconhecimento dos profissionais em como realizar a SAE e o PE no contexto hospitalar, especialmente pela equipe de técnicos de enfermagem, também surge como uma fragilidade apontada pelos enfermeiros entrevistados, como pode ser visualizado nos seguintes relatos: "As principais fragilidades ocorrem pelo desconhecimento da SAE, implementação de maneira errônea, sem base cientifica. Além disso, a falta de treinamento dos técnicos de enfermagem para seguimento dos cuidados conforme prescrição" (E5). "Vejo como fragilidade 0 desconhecimento de como implementar o processo" (E6). "Percebo como fragilidade a organização que está voltada para a questão burocrática e a falta de reconhecimento da sua importância pela equipe" (E8). "Existe uma qualificação limitada e um déficit de entendimento dos profissionais sobre a importância do processo. Observo que é uma prática pouco entendida tanto pelos profissionais que utilizam, quanto para os que devem desenvolver" (E10). "As fragilidades envolvem a utilização/conhecimento NIC e NOC no serviço em si, pois nós enfermeiros conhecemos o processo de enfermagem, mas os técnicos na maioria não, o que dificulta a aplicação e realização do mesmo" (E14). "Sim, acho que existem fragilidades no processo de aplicação, falta conhecimento de fisiologia, anatomia e de gestão para alguns enfermeiros" (E15).

Embora a maioria dos relatos refira-se ao déficit de conhecimentos dos técnicos de enfermagem sobre O PE e sobre a SAE, alguns participantes referiram-se também às fragilidades dos enfermeiros sobre esse aspecto. Nesse sentido, E 15 atribui essa fragilidade a ausência de conhecimento de fisiologia, anatomia e de gestão visualizada em alguns enfermeiros.

\section{Fragmentação do processo e fragilidades dos instrumentos}

O processo acaba sendo visto de maneira fragmentada, pois alguns enfermeiros relatam que o mesmo não engloba as necessidades do paciente de maneira esclarecedora, havendo também uma falha em sua implementação, pois muitos profissionais ainda o vivenciam como uma obrigação a ser realizada no serviço: "percebo como fragilidade a fragmentação do processo" (E1). "Nem sempre o instrumento engloba as necessidades do indivíduo" (E12). "Deixa a desejar, pois ainda não temos de forma sistematizada" (E3). "Percebo ainda um pouco fragmentada, como se fosse uma obrigação sem sentido para muitos" (E1). "Percebo uma falha no processo, pois nem sempre são realizadas todas 
as etapas. Vejo a utilização do histórico e prescrição, porém não se aplica diagnósticos e avaliação com base nas literaturas de NANDA e NOC, na maioria das vezes. Contudo, já está sendo inserido no cotidiano do serviço" (E5).

A terceira unidade de base apresentou as estratégias sugeridas para auxiliar na realização do PE. Nessa unidade, agruparam-se duas categorias: capacitação/sensibilização para os profissionais; maior integração entre os profissionais e adaptação/reavaliação do processo e dos instrumentos.

\section{Capacitação/sensibilização para os profissionais}

Como uma das estratégias sugeridas pelos enfermeiros entrevistados para auxiliar a melhor realização do PE no contexto hospitalar, surge a capacitação e sensibilização da equipe de profissionais, trazendo a importância das orientações corretas e uma explanação mais aprofundada acerca do mesmo, também disponibilizando treinamento para a equipe: "Viabilização e facilitação do processo de enfermagem, realização de cursos, incentivo à busca de conhecimentos e a valorização de todo grupo" (E1). "Capacitações, com orientações da importância da realização do processo" (E4). "Penso que seria interessante a capacitação profissional, mais aprofundada sobre Processo de Enfermagem e treinamento de novos colaboradores, recém-admitidos no serviço" (E5). "Intensificação e melhores explanações de como funciona o processo e de como utiliza-lo na prática" (E6). "Organização entre profissionais envolvidos, capacitação para todos os profissionais da importância, organização da entrada e atendimento ao paciente, equipe adequada" (E7). "Treinamento dos profissionais para que a equipe reconheça a importância do Processo de Enfermagem [...]" (E8). "Treinamento individualizado dos profissionais para cada setor mostrando sua importância e a oferta de mais qualificação" (E10).

Os enfermeiros sentem a necessidade de qualificação da equipe no que se refere tanto aos aspectos teóricos e de compreensão da importância do desenvolvimento do PE, quanto de como efetivamente desenvolvê-lo na prática.

\section{Maior integração entre os profissionais e Adaptação/reavaliação do processo e dos instrumentos}

A maior integração entre os profissionais também emerge como uma estratégia, visando envolver a equipe toda sobre a importância da realização correta do $\mathrm{PE}$, para obter um cuidado eficiente e de qualidade com o paciente que está internado: "Melhorar a relação entre a equipe; conscientizar sobre a importância do PE para o cuidado" (E3). "[...] Maior tempo para realização do mesmo e um trabalho integrado" (E8). "Acredito que integrar mais a equipe ao processo, pontuando sua real importância para um cuidado mais satisfatório, objetivando a redução da internação hospitalar e possíveis complicações" (E9).

Os enfermeiros sugerem tanto na integração entre os pares, ou seja, os profissionais, quanto destes com o PE para que compreendam a sua importância para o cuidado. Referiram, ainda, o interesse na adaptação e reavaliação no modo de realizar o $\mathrm{PE}$, pois o mesmo é visto como de difícil acesso e entendimento por parte da equipe, o que acaba dificultando a sua realização: "Adaptação do instrumento para a realidade do serviço" (E12). "O processo poderia ser mais simplificado e de fácil acesso/entendimento no serviço" (E14). Observa-se nos relatos dos enfermeiros que ao serem adaptados para a realidade do serviço e planejados de forma mais simples, os instrumentos e o PE poderiam ter um acesso e entendimento diferenciado por parte dos profissionais envolvidos.

\section{DISCUSSÃO}

Os participantes da presente pesquisa, elegeram algumas potencialidades vivenciadas referente ao PE. Uma delas, diz respeito a contribuição do PE para a avaliação do paciente de acordo com a sua singularidade. Esse dado é semelhante ao encontrado em um estudo que objetivou identificar a existência da aplicação da SAE nos hospitais dentre suas dificuldades, facilitar no entendimento da implementação da SAE a fim de auxiliar na gerência de funcionários em um ambiente hospitalar ${ }^{(11)}$. No estudo em questão, os autores descrevem que o PE proporciona organização, um atendimento individualizado, humanizado, com qualidade e de maneira integral, passando a observar o paciente como um todo, em sua singularidade ${ }^{(11)}$.

Os enfermeiros na presente pesquisa, referiram ainda que $O$ PE contribui para $O$ fortalecimento do vínculo entre o profissional e o paciente, por meio das ações que são voltadas ao ser humano e não à doença.

Nesse sentido, salienta-se os diagnósticos de enfermagem, que diferente de outros diagnósticos, não objetiva a investigação e descrição de uma doença, mas sim, a resposta humana aos problemas de saúde atuais ou 
potências e processos de vida do ser humano, da família ou da comunidade, proporcionando embasamento para elaborar as intervenções de enfermagem de forma a alcançar resultados pelos quais o enfermeiro é responsável ${ }^{(8)}$. Por essa razão, o diagnóstico de enfermagem é considerado a etapa mais complexa do processo de enfermagem, constituindo-se em importante desafio para o enfermeiro por requerer dele raciocínio clínico e pensamento crítico para interpretação dos dados obtidos durante anamnese e no exame físico ${ }^{(12)}$.

Além disso, os enfermeiros da presente pesquisa referiram que $\mathrm{O} P \mathrm{PE}$ também contribui para potencializar a qualificação da gestão e a organização do processo de trabalho e de cuidado, bem como à auditoria do serviço. Dados semelhantes foram evidenciados em diferentes estudos que apresentam que a SAE contribui para a organização do serviço, bem como à melhoria na qualidade da assistência, ${ }^{(13-15)}$ proporciona maior eficiência no controle de gastos, além de ser um excelente instrumento para avaliação e fiscalização da assistência(16).

Um participante apresentou a sua compreensão, diferenciando a SAE do $\mathrm{PE}$, referindo-se a SAE com o aspecto organizacional do enfermeiro para que o cuidado possa ocorrer. Já, o PE ele compreendia como a elaboração dos cuidados. Esse dado vem ao encontro do que apresenta a Resolução 358/2009 do COFEN, pois a mesma descreve que a SAE organiza o trabalho profissional quanto ao método, pessoal e instrumentos, tornando possível a operacionalização do $\mathrm{PE}$, que por sua vez, é considerado um instrumento metodológico que orienta o cuidado profissional de Enfermagem e a documentação da prática profissional ${ }^{(3)}$.

Assim, compreende-se que a SAE se aplica tanto no que se refere aos aspectos administrativos, assistenciais, de ensino e de pesquisa em enfermagem. Percebe-se, dessa forma, que a SAE é distinta e mais ampla que PE, pois ela pressupõe uma organização geral do setor de trabalho, como por exemplo: dimensionamento de pessoal, as escalas de trabalho, a distribuição de atividades, a organização de instrumentos, como os protocolos do setor, os manuais, Procedimentos Operacionais Padrão (POPs), normas e as rotinas. Já o PE indica um trabalho profissional específico e pressupõe uma série de atividades interrelacionadas; em outras palavras, indica a utilização de método para organizar a prática clínico-assistencial de cuidados.

Além da qualificação do cuidado e da gestão, o PE contribui para o empoderamento e à autonomia profissional do enfermeiro, conforme descrito pelos participantes da presente pesquisa. Esse dado corrobora com o evidenciado em um estudo desenvolvido com 22 enfermeiros que trabalham nas Unidades Básicas de Saúde (UBS) do Distrito Sanitário Pampulha, em Belo Horizonte, Minas Gerais, com objetivo de identificar os obstáculos apontados por enfermeiros para a implementação do PE em UBS, onde os autores descrevem que O PE valida a prática profissional de toda a equipe de enfermagem, o que define o exercício da profissão e confere mais autonomia aos enfermeiros durante sua realização ${ }^{(17)}$. Considerase, dessa forma, que a aplicabilidade e o conhecimento correto do PE contribuem cada vez mais para o enfermeiro conseguir ter um reconhecimento frente a sua profissão, pois dessa maneira consegue ser visualizado como profissional que avalia, realiza diagnósticos e prescreve cuidados baseado em conhecimento técnico-cientifico, tendo a autonomia durante a sua realização.

Contudo, os participantes da presenta pesquisa, também elencaram algumas fragilidades, dentre elas à rotina intensa, a grande demanda e a sobrecarga de trabalho dos profissionais/equipe de enfermagem, o que dificulta a realização do PE com todas as etapas. Dado semelhante foi evidenciado em pesquisa realizada em um hospital geral da fronteira Oeste do RS, momento em que os participantes referiram a sobrecarga de trabalho dos enfermeiros, a presença de condições inadequadas de trabalho ${ }^{(18)}$. Esse dado é relevante, pois a sobrecarga, somada à longa jornada de trabalho, pode acarretar em fragilidades na habilidade funcional e moral dos enfermeiros/técnicos de enfermagem, ocorrendo dificuldade na elaboração de um trabalho efetivo, como a não realização da SAE e do PE corretamente, o que fragiliza assistência de qualidade ao paciente ${ }^{(19)}$.

Os enfermeiros, participantes da presente pesquisa referem ainda a falta empenho de alguns profissionais para a realização da SAE e que o déficit de funcionários e de materiais, somados a outras fragilidades, impossibilitam o desenvolvimento das ações planejadas. Esse dado é corroborado por estudo realizado em um hospital público no Amapá, no qual os autores 
descrevem que o enfermeiro tem enfrentado dificuldades na implementação da SAE como instrumento científico de trabalho(20). Essa dificuldade, pode estar atrelada às condições de trabalho, ao quantitativo insuficiente de funcionários e à formação dos enfermeiros com pouco estimulo para valorização da aplicabilidade da SAE ${ }^{(21)}$

Outra fragilidade evidenciada na presente pesquisa refere-se ao desconhecimento dos profissionais em como realizar a SAE e o PE no contexto hospitalar, especialmente pela equipe de técnicos de enfermagem. Estudo desenvolvido com objetivo de apresentar a percepção e o conhecimento de enfermeiros e acadêmicos de enfermagem brasileiros quanto à SAE demonstrou que os enfermeiros percebem a pouco valorização do PE na prática profissional, ao referirem que durante a formação acadêmica, muitos não compreenderam ou reconheceram o PE como uma metodologia de trabalho(22). Esse dado conduz à reflexão que a formação, seja do enfermeiro ou do técnico de enfermagem precisa ser fortalecida no aspecto do ensino e da aplicação da SAE e do PE com vistas a auxiliar na compreensão, conhecimento e sensibilização dos profissionais para a sua relevância no contexto de cuidados.

A esse respeito, para uma efetiva implantação da SAE e do PE, é necessário o comprometimento da gestão de enfermagem com a proposta, por meio de reuniões e no planejamento de ações, incluindo a sensibilização de toda a equipe para a importância dessa metodologia(12). Tanto a SAE como o PE devem ser reconhecidos por toda a equipe, com o delineamento da importância que todos saibam o motivo de ser aplicado de forma correta, pois sua utilização promove um cuidado sistematizado frente ao paciente internado.

A efetivação do PE como uma obrigatoriedade a ser realizada e não pela sua relevância também foi evidenciado, na presente pesquisa, como uma fragilidade. A resolução 358/2009 deixa evidente que a SAE e o PE devem ser realizados de maneira obrigatória em todos os cenários onde ocorre o cuidado profissional de enfermagem, tornando dessa forma essencial para um cuidado organizado dentro do serviço de saúde ${ }^{(3)}$. Contudo, quando o cuidado é realizado somente por obrigação e de maneira reproduzida, acaba tornando-se mecanizado, e sua utilização não contribui à obtenção dos resultados que se esperam, mas sim para cumprir uma legislação, o que acarreta a falta de interesse e sensibilização dos profissionais para realiza-lo de maneira correta $^{(23)}$.

Assim, evidencia-se que, para além de uma obrigatoriedade, os profissionais de enfermagem devem compreender a sua necessidade e relevância no contexto clínico-assistencial, com vistas para o melhor cuidado as pessoas, pois ao se utilizar o PE, trabalha-se com evidências e, dessa forma, contribui-se também para a valorização da categoria profissional.

Na presente pesquisa, o PE foi mencionado como de difícil acesso e entendimento por parte da equipe, o que dificulta a sua realização no serviço. Para tanto, os profissionais sugeriram a sua reavaliação e adaptação para o cenário. Em uma Revisão Integrativa da Literatura que objetivou Identificar nas produções científicas o impacto decorrente do uso das tecnologias de informática na implementação da SAE em serviços de saúde hospitalares, também evidenciou-se fragilidades em relação a informatização do PE, visto que alguns profissionais sentiam dificuldade em realizá-lo, o que acabava por trazer a necessidade de uma readaptação para que se efetivasse melhorias no sistema operacional ${ }^{(24)}$.

Além disso, o estudo sugere a necessidade da realização de um treinamento com os enfermeiros sobre a sua operacionalização e o seu desenvolvimento(25). Assim, salienta-se a relevância da implementação da Política Nacional de Educação Permanente, a fim de contribuir para o fortalecimento do ensino sobre a temática e troca de experiências entre os profissionais das instituições.

Como estratégias sugeridas pelos enfermeiros para auxiliar na superação das fragilidades vivenciadas, encontra-se a capacitação e sensibilização da equipe. Dessa maneira, destaca-se a necessidade de uma educação permanente no serviço, por meio de treinamentos e capacitações para toda a equipe de enfermagem acerca do PE, ressaltando como o mesmo possibilita uma assistência organizada, efetiva e de qualidade para o paciente em sua individualidade ${ }^{(17)}$.

A maior integração entre os profissionais também emerge como uma estratégia, sensibilizando toda a equipe sobre a importância da realização correta do PE para obter um cuidado eficiente e de qualidade para o paciente internado. A complementação do trabalho em equipe está relacionada com a multidisciplinaridade, na qual a integração de toda a equipe se articula e se complementa visando a recuperação do paciente ${ }^{(23)}$. Dessa 
forma, todo o cuidado desenvolvido pelos profissionais de acordo com as atividades privativas de sua profissão, é direcionado a melhora do paciente, focando na sua recuperação e na diminuição do tempo de internação do mesmo.

\section{CONSIDERAÇÕES FINAIS}

A realização dessa pesquisa foi satisfatória, pois possibilitou 0 atendimento do objetivo proposto, uma vez que permitiu compreender a vivência de enfermeiros, atuantes no contexto hospitalar acerca do Processo de Enfermagem, por meio das potencialidades e fragilidades vivenciadas, bem como das estratégias sugeridas para auxiliar nas fragilidades.

Como principais resultados evidenciaram-se as potencialidades do PE no contexto pesquisado: qualificação da avaliação, singularização, cuidado e segurança do paciente; qualificação da gestão e organização do processo e cuidado/trabalho; e o empoderamento e autonomia profissional. Como fragilidades, a demanda/sobrecarga de trabalho dos profissionais; o desconhecimento dos profissionais sobre a SAE e o PE e a fragmentação do processo e fragilidades dos instrumentos. As estratégias citadas pelos profissionais sugeriram o desenvolvimento de capacitação/sensibilização para os profissionais; maior integração entre os profissionais e a adaptação e reavaliação do processo e instrumentos.

As contribuições dessa pesquisa para a enfermagem como ciência, se dá pelo fato de que os dados obtidos podem ser utilizados em diferentes contextos, serem comparados a de outros estudos e ainda, estimular pesquisas semelhantes em outros cenários e com diferentes públicos. Já as contribuições para a prática, o fato de os enfermeiros refletirem sobre o seu cotidiano, por meio das potencialidades e fragilidades já os movimenta à busca de maior conhecimento e para possíveis estratégias de qualificação profissional, seja ela de maneira individual ou coletiva junto aos pares.

Como limitação dessa pesquisa, compreende-se o fato de ter sido desenvolvida apenas com os enfermeiros, não contemplando a equipe de técnicos de enfermagem, os quais são fundamentais para o desenvolvimento do PE. Contudo, compreende-se que o primeiro passo para o sucesso na utilização é a sensibilização/reflexão dos líderes das equipes, ou seja, os enfermeiros para posteriormente ser ampliada a proposta aos técnicos de enfermagem.

\section{REFERÊNCIAS}

1. Marinelli NP, Silva ARA, Silva DNO. Sistematização da Assistência de Enfermagem: Desafios para implantação. Revista Enfermagem Contemporânea. 2015;4(2):254-63. DOI: $10.17267 / 2317-$ 3378rec.v4i2.523.

2. Santos MG, Silva TG, Frizon G, Quinto AS. Etapas do processo de enfermagem: uma revisão narrativa. Enferm. Foco. 2017;8(4):49-53. Disponível em: http://revista.cofen.gov.br/index.php/enferm agem/article/view/1032/416.

3. Conselho Federal de Enfermagem (COFEN). Resolução COFEN- 358/2009. Dispõe sobre a Sistematização da Assistência de Enfermagem e a implementação do Processo de Enfermagem. 2009 [acesso em: 10 de dez. 2020]. Disponível em: http://www.cofen.gov.br/resoluo-cofen3582009 4384.html.

4. Meneguin S, Banja PFT, Ferreira MLS. Care for hospitalized elderly patients: implications for nursing team. Rev. Enferm. UERJ. 2017;25:e16107.

DOI: 10.12957/reuerj.2017.16107.

5. Neves L, Gondim AA, Soares SCMR, Coelho $D P$, Pinheiro JAM. The impact of the hospitalization process on the caregiver of a chronic critical patient hospitalized in a SemiIntensive Care Unit. Esc. Anna Nery Rev. Enferm. 2018;22(2):e20170304. DOI: 10.1590/2177-9465-ean-2017-0304.

6. Santos RCA, Miranda FAN. Importância do vínculo entre profissional-usuário na estratégia de saúde da família. Rev. Enferm. UFSM. 2016;6(3):350-9. DOI: $\underline{10.5902 / 2179769217313 .}$

7. Ferreira LB, Oliveira JSA, Gonçalves RG, Elias TMN, Medeiros SM, Mororó DDS. Cuidar de enfermagem às famílias de crianças e adolescentes hospitalizados. Rev. Enferm. UFPE. 2019;13(1):23-31. DOI: 10.5205/19818963-v13i1a237672p23-31-2019.

8. Diagnósticos definições e classificações de enfermagem da NANDA: 2018-2020. 11aㅡ ed. 
Porto Alegre: Artme; 2018.

9. Moraes R, Galiazzi MC. Análise textual discursiva. $2^{\text {a }}$ ed. Editora Unijuí; 2011.

10. Brasil. Ministério da Saúde. Portaria no 466 de 12 de dezembro de 2012. Disponível em: http://bvsms.saude.gov.br/bvs/saudelegis/cn s/2013/res0466 1212 2012.html.

11. Silva MC, Macedo JS, Oliveira LP, Sandim LS. A implementação da sistematização da assistência de enfermagem no ambiente hospitalar. Braz. J. Of Develop. 2020;6(6):33293-306.

DOI: 10.34117/bjdv6n6-039.

12. Ubaldo I, Matos E, Salum NC. Diagnósticos de enfermagem da Nanda-I com base nos problemas segundo teoria de Wanda Horta. Cogitare Enferm. 2015;20(4):687-94. Disponível em: http://www.redalyc.org/articulo.oa?id=4836 47681006.

13. Riegel $F$, Oliveira Jr NJ. Nursing process: implications for the safety of surgical patients. Cogitare Enferm. 2017;22(4):1-5. DOI: $\underline{10.5380 / c e . v 22 i 1.45577 .}$.

14. Pereira GN, Abreu RNDC, Bonfim IM, Rodrigues AMU, Monteiro LB, Sobrinho JM. Relação entre sistematização da assistência de enfermagem e segurança do paciente. Enferm Foco. 2017;8(2):21-5. DOI: 10.21675/2357-707X.2017.v8.n2.985.

15. Bandin M, Toledo VP, Garcia APRF. Contribution of transference to the psychiatric nursing process. Rev Bras Enferm [Internet]. 2018;71(Suppl 5):2161-8. DOI: 10.1590/0034-7167-2016-0640.

16. Silva RS, Almeida ARLP, Oliveira FA, Oliveira AS, Sampaio MRFB, Paixão GPN. Sistematização da Assistência de Enfermagem na perspectiva da equipe. Enferm. Foco. 2016;7(2):32-6. Disponível em: http://revista.cofen.gov.br/index.php/enferm agem/article/view/803/328.

17. Pires DBP, Teixeira DJ. Processo de Enfermagem: obstáculos apontados por enfermeiros em unidades básicas de saúde de Belo Horizonte. Enferm. Rev. 2020;23(1). Disponível http://periodicos.pucminas.br/index.php/enf ermagemrevista/article/view/23633.

18. Xavier LF, Silva SBM, Oliveira OD, Nazario YCOS, Morais Jr SLA. Sistematização da assistência de enfermagem: o conhecimento de enfermeiros do município de Ji-Paraná, Rondônia, Brasil. Nursing. 2018;21(239):2110-3. Disponível em: http://www.revistanursing.com.br/revistas/2 39-

Abril2018/sistematizacao_assistencia de enf ermagem.pdf.

19. Costa CS, Normann KAS, Tanaka AKSR, Cicolella DA. A influência da sobrecarga de trabalho do enfermeiro na qualidade da assistência. Rev. UNINGÁ. 2018;55(4):110-20. Disponível em: http://revista.uninga.br/index.php/uninga/ar ticle/view/2403/1796.

20. Tavares FMM, Tavares WS. Elaboração do instrumento de sistematização da assistência de enfermagem: relato de experiência. $R$ Enferm Cent O Min. 2018;8:e2015. DOI: 10.19175/recom.v7i0.2015.

21. Gutiérrez MGR, Morais SCRV. Sistematização da assistência de enfermagem e a formação da identidade profissional. Rev. Bras. Enferm. 2017;70(2):455-60. DOI: 10.1590/0034-71672016-0515.

22. Oliveira MR, Almeida PC, Moreira TMM, Torres RAM. Nursing care systematization: perceptions and knowledge of the Brazilian nursing. Rev Bras Enferm. 2019;72(6):154753. DOI: 10.1590/0034-7167-2018-0606.

23. Dotto II, Backes DS, Dalcin CB, Lunardi Filho WD, Siqueira $\mathrm{HCH}$, Zamberlan C. Systematization of nursing assistance: order, disorder or (re) organization? Rev. Enferm. UFPE online. 2017;11(10):3821-9. DOI: $\underline{10.5205 / 1981-8963-v 11 i 10 a 25235 p 3821-}$ 3829-2017.

24. Pissaia LF, Costa AEK, Moreschi C, Rempel C, Correno I, Granada D. Impacto de tecnologias na implementação da sistematização da assistência de enfermagem hospitalar: uma revisão integrativa. Revista de Epidemiologia e Controle de Infecção. 2018;8(1):92-100. DOI: 10.17058/reci.v1i1.8953. 
12| Moreira RF, Ha a BK, Zamberlan C, et al.

25. Alencar IGM, Nunes VS, Alves AS, Let $S$. Implementação e implantação da sistematização da assistência de enfermagem. Rev Enferm UFPE online. 2018;12(4):1174-8. DOI: $10.5205 / 1981-8963-$ v12i4a231030p1174-1178-2018.

\section{Editores Responsáveis:}

Patrícia Pinto Braga Angélica Mônica Andrade

Nota: Não houve financiamento de agência de fomento.

Recebido em: 08/04/2021

Aprovado em: 18/11/2021 